\title{
Role of Cerebellum in Adaptive Modification of Reflex Blinks
}

\author{
John J. Pellegrini ${ }^{1}$ and Craig Evinger ${ }^{2,3}$ \\ ${ }^{1}$ Department of Biology \\ The College of St. Catherine \\ St. Paul, Minnesota 55105 \\ ${ }^{2}$ Departments of Neurobiology and Behavior and Ophthalmology \\ State University of New York at Stony Brook \\ Stony Brook, New York 11794-5230
}

\begin{abstract}
We investigated the involvement of the cerebellar cortex in the adaptive modification of corneal reflex blinks and the regulation of normal trigeminal reflex blinks in rats. The ansiform Crus I region contained blink-related Purkinje cells that exhibited a complex spike $20.4 \mathrm{msec}$ after a corneal stimulus and a burst of simple spike activity correlated with the termination of orbicularis oculi activity. This occurrence of the complex spike correlated with trigeminal sensory information associated with the blink-evoking stimulus, and the burst of simple spike activity correlated with sensory feedback about the occurrence of a blink. Inactivation of the inferior olive with lidocaine prevented all complex and significantly reduced simple spike modulation of blink-related Purkinje cells, but did not alter orbicularis oculi activity evoked by corneal stimulation. In contrast, both acute and chronic lesions of the cerebellar cortex containing blink-related Purkinje cells blocked adaptive increases in orbicularis oculi activity of the lid ipsilateral but not contralateral to the lesion. These data are consistent with the hypothesis that the cerebellum is part of a trigeminal reflex blink circuit. Changes in trigeminal signals produce modifications of the cerebellar cortex, which in turn, reinforce or stabilize modifications of brainstem blink circuits. When the trigeminal system does not attempt to alter the magnitude of trigeminal
\end{abstract}

${ }^{3}$ Corresponding author. reflex blinks, cerebellar input has little or no effect on reflex blinks.

\section{Introduction}

Reflex blinks are a simple motor behavior that undergo rapid adaptive modification in response to altered sensory feedback (Evinger and Manning 1988; Evinger et al. 1989; Schicatano et al. 1996). For example, reducing the motility of the upper eyelid initiates a rapid increase in the magnitude of the lid closing, orbicularis oculi (OO) muscle activity to compensate for the reduction in lid mobility. This adaptive behavior is a learned response rather than a proprioceptive reaction to restriction of lid motion, because the larger than normal OO activity elicited by a blink-evoking stimulus remains for a period after returning lid motility to normal. Blink adaptation can also reduce the drive on blink circuits. Assisting lid closure causes a learned reduction in $\mathrm{OO}$ activity in response to a blink-evoking stimulus. Therefore, the nervous system must constantly compare the neural representation of the motor command for a blink with the proprioceptive feedback from the actual reflex blink. A consistent difference between these two values initiates an active modification of the trigeminal drive on $\mathrm{OO}$ motoneurons to maintain a constant relationship between stimulus and blink magnitude. Recent evidence indicates that these adaptive modifications occur in the spinal trigeminal complex (Schicatano et al. 1996).

Adaptive blink mechanisms can have a significant role in disease. For example, Bell's palsy, resulting from unilateral facial nerve damage, induces changes in trigeminal blink circuits identical to those generated by upper eyelid restraint (Huffman et al. 1996; Schicatano et al. 1996). Such adap-

LEARNING \& MEMORY 3:77-87 @ 1997 by Cold Spring Harbor Laboratory Press ISSN1072-0502/97 \$5.00

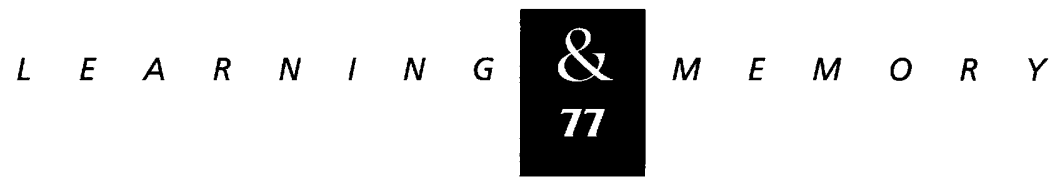


tive modifications assist in compensating for the reduction in lid motility caused by facial nerve damage. Modifications of trigeminal blink circuits initiated by adaptive processes can also cause disease states. Under conditions of decreased inhibition of the trigeminal system, a normally adaptive increase in trigeminal reflex blink excitability can lead to uncontrollable spasms of lid closure, or blepharospasm (Chuke et al. 1996; Schicatano et al. 1997). Therefore, investigating the neural mechanisms underlying adaptive modification of reflex blinks is important for understanding disease processes, as well as for understanding basic mechanisms underlying procedural learning.

The importance of the cerebellum in adaptive modification of the vestibulo-ocular reflex (for review, see dulac et al. 1995) and in conditioned learning of the blink reflex (for review, see Thompson and Krupa 1994) suggests that the cerebellum has a role in adaptive modification of trigeminal reflex blinks. One can envision at least two roles for the cerebellum in adaptive modification of the reflex blinks. Using trigeminal mossy and climbing fiber inputs, the cerebellum could detect errors between the expected and actual blink magnitude. The cerebellum would then actively regulate the magnitude of trigeminal reflex blinks to eliminate discrepancies between an efferent copy signal of the blink and the actual blink. In this scheme, the cerebellum is a regulatory element acting on an autonomous reflex blink circuit. A second possibility is that the cerebellum is an element of the reflex blink circuit. In this scheme, the cerebellum receives trigeminal sensory information and uses this information to reinforce and stabilize modifications initiated within trigeminal circuits. In contrast to the first scheme, the interactions between the cerebellum and the trigeminal system are reciprocal in this second model. The current study reports on the discharge characteristics of blinkrelated Purkinje cells and explores their role in normal reflex blinks and reflex blink adaptation. The data indicate that the trigeminal system and the cerebellum exert reciprocal effects when the nervous system adapts the gain-of-trigeminal reflex blinks.

\section{Materials and Methods}

\section{PROCEDURES}

Subjects for all experiments were male Sprague-Dawley rats. The details of the procedures used in this study have been reported in previous studies (Evinger et al. 1993; Basso et al. 1996). The animals were prepared for recording electromyograph (EMG) activity of the lid closing, orbicularis oculi muscle (OOemg) and single-unit, extracellular recording. Two preparations were employed. In initial experiments, rats were anesthetized with ketamine $(90 \mathrm{mg} / \mathrm{kg})$ and xylazine $(10 \mathrm{mg} / \mathrm{kg})$ and then underwent precollicular decerebration by aspiration. No further anesthesia was administered. In later experiments, rats were anesthetized with urethane [15 grams/ $\mathrm{kg}$ in phosphate buffer, $\mathrm{pH}$ 7.4 , intraperitoneally (i.p.)] and no decerebration was performed. To record OOemg, a pair of singlestranded, Teflon-coated, stainless steel wires were implanted into the medial and lateral margins of both the right and left eyelids. To allow restraint of the eyelid, a silk suture was attached to the center of the lower margin of both upper eyelids. To provide access to the cerebellum for microelectrodes, the bone overlying the Crus I region of the cerebellum was removed. In some experiments, a hole was drilled in the skull overlying the inferior olive to allow access to this region with microelectrodes. In other experiments, either an acute or chronic unilateral lesion of the Crus I region was performed by aspiration. For chronic lesions, the rat received general anesthesia (ketamine, $90 \mathrm{mg}$ / $\mathrm{kg}$; xylazine, $10 \mathrm{mg} / \mathrm{kg}$ ). Using aseptic techniques, the skull overlying the left Crus I region was removed and the underlying cerebellar cortex was aspirated. The bone defect was covered with bone wax and the overlying skin sutured. Rats were allowed to recover from anesthesia and treated with analgesics as necessary. Three or four days later, rats were reanesthetized and prepared as described above. For acute lesions, an aspiration of Crus I cerebellar cortex was performed during the experiment. At the end of all experiments, the rats were perfused with $6 \%$ dextran to wash out blood, followed by $10 \%$ formalin to allow histological examination of lesions or location of fast green dye injections.

Blinks were evoked by electrical stimulation of the cornea through a pair of silver ball electrodes. In most cases, a single $100-\mu$ sec stimulus elicited a corneal reflex blink. In other cases, it was necessary to present three $100-\mu$ sec stimuli to evoke a corneal reflex blink. To test the excitability of corneal reflex blinks, pairs of identical stimuli with a 75-msec interstimulus interval were presented, the paired stimulus paradigm (Pellegrini and Evinger 1995). To avoid habituation, the inter-

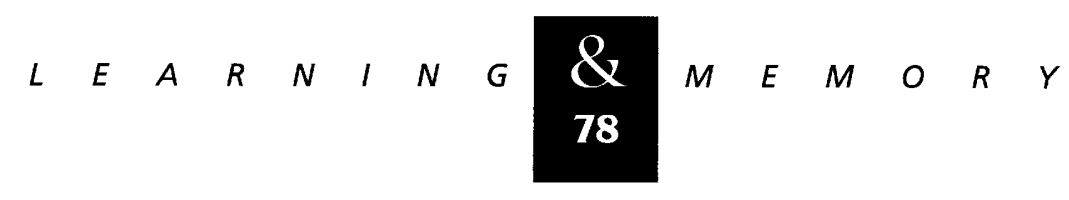


CEREBELLUM IN REFLEX BLINK ADAPTATION

val between stimuli (or pairs of stimuli) was $45 \pm 5$ sec.

To induce adaptive increases in the drive to orbicularis oculi motoneurons, the upper eyelids were restrained by connecting the suture attached to the upper eyelid to a fixed bar. Identical corneal stimuli were presented before and after lid restraint. Both the left and right cornea received stimuli at the same time.

Single Purkinje cells and interpositus neurons were recorded extracellularly with glass micropipettes filled with $2 \mathrm{M} \mathrm{NaCl}$ saturated with fast green. The signals were filtered from 1 to $10 \mathrm{kHz}$. Purkinje cells were identified by their characteristic complex spike activity. To anesthetize the inferior olive, we used double-barreled electrodes. One barrel was filled with $2 \%$ lidocaine and the other barrel was filled with $2 \mathrm{M} \mathrm{NaCl}$ saturated with fast green. The lidocaine barrel was attached to a Picospritzer (General Valve Co.) to allow brief microinjections of lidocaine. To determine the efficacy of inferior olive inactivation, we simultaneously recorded from blink-related Purkinje cells during corneal stimulation. We considered the inferior olive inactivated, when corneal stimulation no longer elicited complex spikes in Purkinje cells, but the OOemg activity of corneal-evoked blinks was still present. Fast green dye injections (Thomas and Wilson 1965) were made at the site of Purkinje cell recordings and lidocaine injections.

\section{DATA COLLECTION AND ANALYSIS}

OOemg, single-unit activity, and the stimuus were stored on analog tape for later off-line analysis. OOemg data were digitized ( $5 \mathrm{kHz}$ per channel, 12-bit precision) and analyzed using laboratorydeveloped software. The OOemg records were rectified and integrated and averaged to determine OOemg magnitude. Individual simple spikes were recognized with a window discriminator and the output of the discriminator was digitized (2 $\mathrm{kHz}, 12$-bit precision). Simple spike analysis used raster displays, histograms (10-msec bins), or spike density profiles. The spike density function is a low-pass-filtered gauge of the probability of spike occurrence over time. Our analysis convolved the spike train with Gaussian kernel, $\sigma=2 \mathrm{msec}$ (for details, see Richmond et al. 1987).

\section{Results}

\section{BLINK-RELATED PURKINJE CELLS}

Purkinje cells whose discharge correlated with corneal stimulation and blinking were found in specific regions of the cerebellar cortex. Blink-related Purkinje cells were found consistently in Crus I of the ansiform lobule (Fig. 1). No systematic search for all possible locations of blink-related Purkinje cells was performed, but blink-related Purkinje cells responding to corneal stimulation were not found in nearby regions.

In response to stimulation of the cornea, blink-
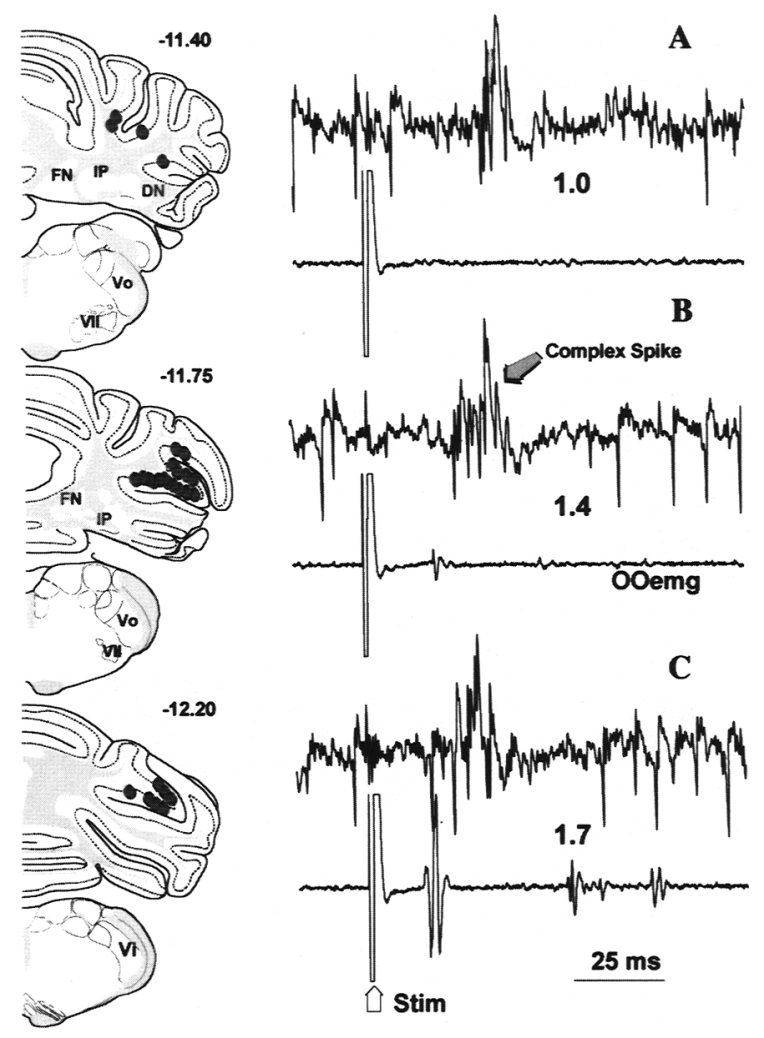

Figure 1: Location and characteristics of blink-related Purkinje cells. (Left) Three schematic sections of rat cerebellum adapted from Swanson (1992) located 11.4, 11.75 , and $12.2 \mathrm{~mm}$ posterior to bregma. Circles show location of blink-related Purkinje cells marked by fast green injections. $(A-C)$ Individual records of Purkinje cell (top trace) and simultaneous orbicularis oculi EMG activity (bottom trace, OOemg) collected at threshold for evoking a complex spike response $1.0(A), 1.4(B)$, and $1.7(C)$ times threshold. (DN) Dentate nucleus; (FN) fastigial nucleus; (IP) interpositus nucleus; (Vi) spinal trigeminal nucleus interpolaris subdivision; (Vo) spinal trigeminal nucleus oralis subdivision; (VII) facial nucleus.

$$
\text { ……‥ }
$$


related Purkinje cells exhibited a complex spike, followed by a brief pause in simple spike activity and a subsequent burst of simple spike activity (Figs. 1 and 2). The complex spike was the most sensitive component of this evoked response. The complex spike could be elicited by corneal stimuli that were too weak to evoke OOemg activity (Fig. 1, cf. A and C). When the corneal stimulation failed to evoke OOemg activity, the pause in simple spike activity was often longer than when a blink occurred. This increased pause duration probably resulted from the absence of a burst of simple spike activity produced by mossy fiber inputs related to the occurrence of a blink (see below). Following a corneal stimulus that evoked a blink, the complex spike occurred after the onset of OOemg activity. The mean latency of complex spike activity was $20.4 \mathrm{msec}( \pm 3.78, n=47$ cells $)$, whereas the mean latency of OOemg activity was only $16.46 \mathrm{msec}$ ( $\pm 5.8 \mathrm{msec}, n=68$ experiments). The burst in simple spike activity typically occurred near or co- incident with the end of the OOemg activity and lasted 50-75 msec (Fig. 2). The mean simple spike frequency for a 50-msec period of the burst divided by the mean simple spike frequency during a 50msec period of spontaneous activity averaged 1.46 $( \pm 0.205, n=18)$. The mean, maximum firing frequency during the burst was 122 spikes/sec $(n=47)$ for all blink-related Purkinje cells. The burst in Purkinje cell simple spike activity with corneal evoked blinks did not result from a rebound excitation following the complex spike. There was always a burst of simple spike discharge following a complex spike evoked by corneal stimulation that elicited a blink, but no increase of simple spike discharge following spontaneous complex spikes (Fig. 3). Therefore, the burst of simple spike discharge with the termination of OOemg activity carried information about the occurrence of a corneally evoked reflex blink. Because the cerebellum receives mossy fiber inputs from the trigeminal complex (vanHam and Yeo 1992),
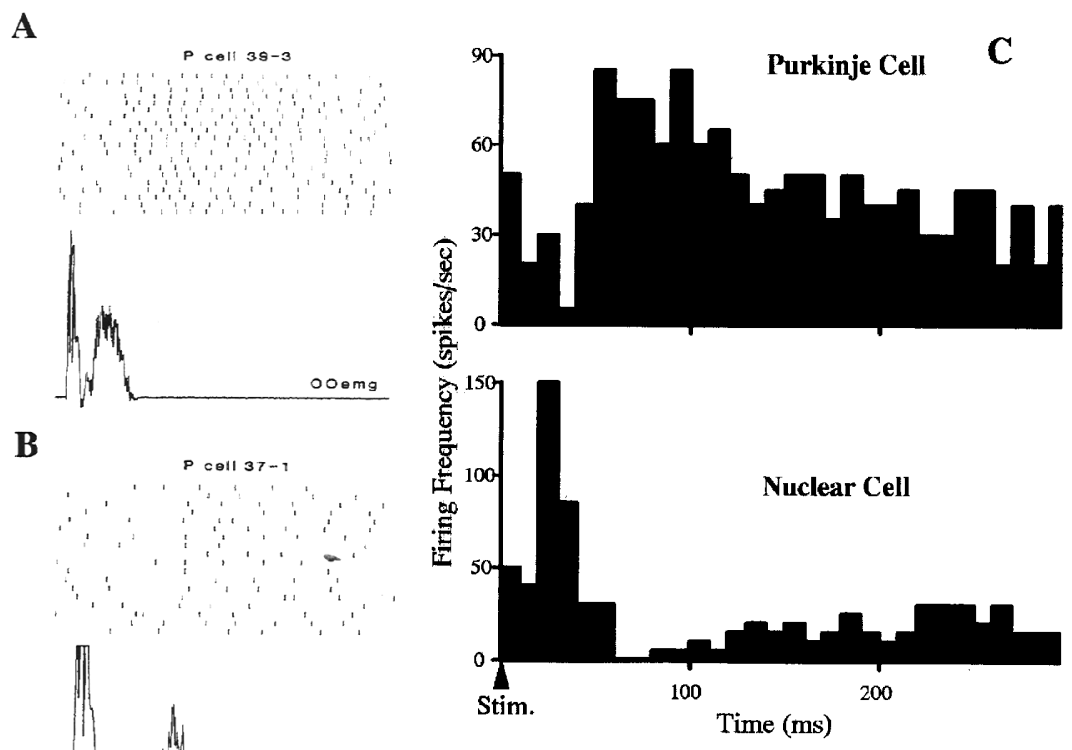

Figure 2: Characteristics of blink-related cerebellar neurons. $(A)$ Raster of simple spike activity of a single blink-related Purkinje cell (39-3) and rectified and averaged OOemg activity of 20 consecutive trials of corneal stimulation. Each mark represents the occurrence of a simple spike and each line of marks is a different trial. The duration of the entire record is $250 \mathrm{msec}$. (B) Raster of simple spike activity of a single blink-related Purkinje cell (37-1) and rectified and averaged OOemg activity of 18 consecutive trials with corneal stimulation. Each mark represents the occurrence of a simple spike and each line of marks is a different trial. The duration of the entire record is $150 \mathrm{msec}$. $(C)$ Histograms of simple spike activity of a blink-related Purkinje cell (top record) and spikes of a blink-related interpositus nucleus neuron (bottom record). Each histogram is the sum of 20 trials using $10-\mathrm{msec}$ bins. The occurrence of the corneal stimulus coincides with the onset of the histogram.

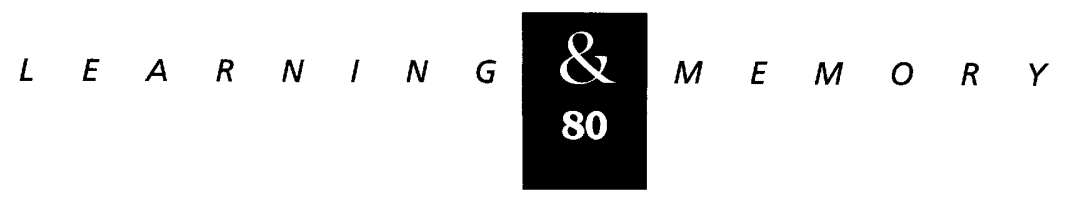




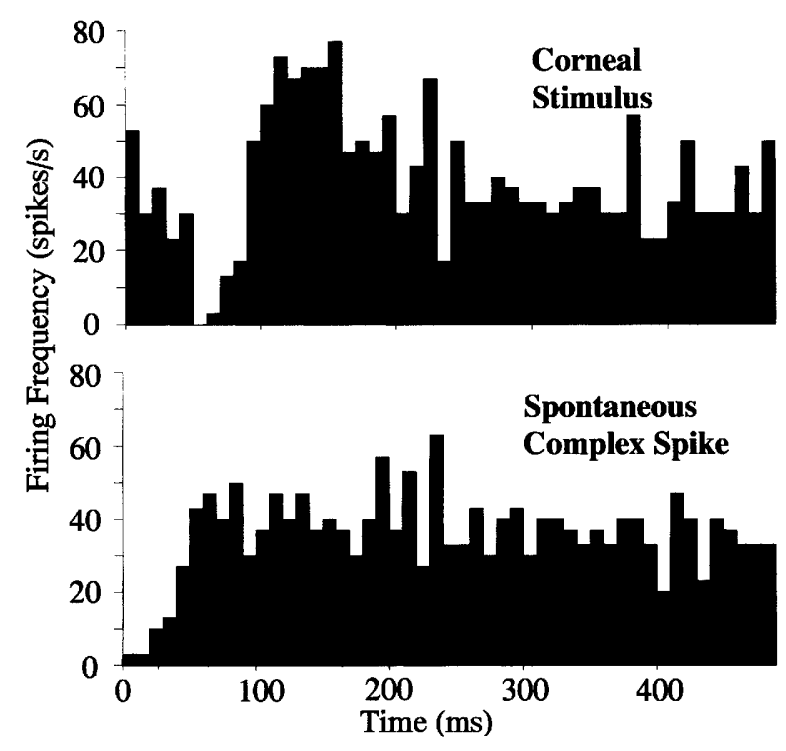

Figure 3: Histograms of simple spikes of blink-related Purkinje cells in response to a corneal stimulus (top record, Corneal Stimulus) and after a spontaneous complex spike (bottom trace, Spontaneous Complex Spike). Each histogram is the sum of 20 trials using 10 -msec bins. The occurrence of the corneal stimulus coincides with the onset of the top histogram. The occurrence of the complex spike coincides with the onset of the bottom histogram.

the burst of simple spike activity probably reflects feedback from the occurrence of a blink.

The Purkinje cell response associated with a corneally evoked reflex blink exhibited three components: (1) a complex spike; (2) a brief pause in simple spike activity; and (3) a burst of simple spike activity associated with the occurrence of a blink. The complex spike occurred after the onset of the blink. The pause in simple spike activity happened during the later components of the OOemg response and the peak burst of simple spike activity followed the termination of OOemg activity (Figs. 1 and 2). Therefore, changes in blinkrelated Purkinje cell activity could only alter the long latency components and termination of the blink. The activity pattern of interpositus neurons with corneal-evoked blinks was a mirror image of blink-related Purkinje cell simple spike discharge (Fig. 2C, lower trace). Just as reported for cat interpositus interneurons (Gruart and Delgado-García 1994), changes in rat interpositus neuronal activity occurs too late to modify anything but the longer latency components of trigeminal reflex blinks.

The activity of blink-related Purkinje cells mirrored the OOemg activity evoked in the paired stimulus paradigm. In all mammalian species tested, the OOemg response to the second of two identical stimuli was less than that to the first stimulus for short interstimulus intervals (Pellegrini and Evinger 1995; Basso et al. 1996; Powers et al. 1997). With these paired corneal stimuli, Purkinje cells exhibited a complex spike in response to the first stimulus, but no complex spike or burst of simple spike discharge in response to the second stimulus (Fig. $4 \mathrm{~A} ; n=18$ cells). The absence of a complex spike to the second stimulus was not attributable to an inability of complex spikes to oc-
A

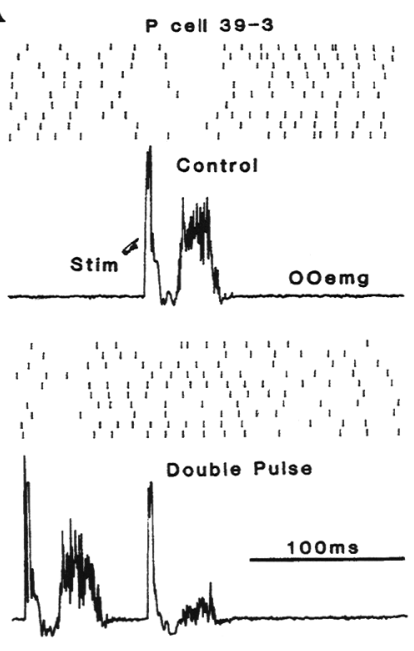

B
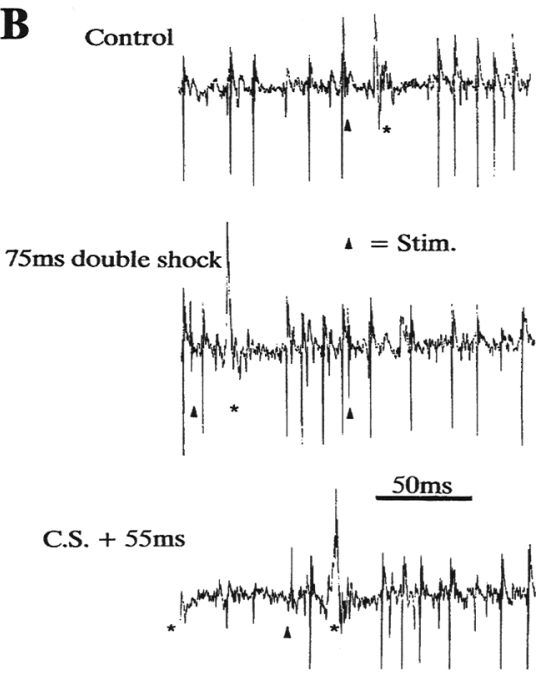

Figure 4: Discharge of blink-related Purkinje cells in response to pairs of corneal stimuli with a 75-msec interstimulus interval. (A) Rasters of simple spike activity and averaged OOemg activity in response to 10 trials with a single corneal stimulus (Stim; Control) and simple spike activity and averaged OOemg activity in response to 10 trials with two corneal stimuli separated by $75 \mathrm{msec}$ (Double Pulse). (B) Individual records of blink-related Purkinje cell activity in response to a single corneal stimulus $(\boldsymbol{\Lambda}$, Control), a pair of corneal stimuli with a 75-msec interstimulus interval (75-msec double shock), and a corneal stimulus presented 55 msec after the occurrence of a spontaneous complex spike (C.S. + 55). Asterisks show the occurrence of the complex spike.

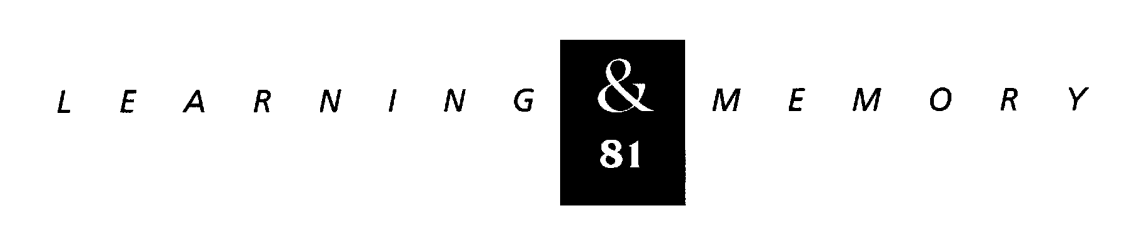


cur 75 msec apart. If a spontaneous complex spike was used to trigger a corneal stimulus $55 \mathrm{msec}$ later, the corneal stimulus evoked a complex spike 75 msec after the spontaneous complex spike and a burst of simple spike activity (Fig. $4 \mathrm{~B} ; n=6$ cells). The absence of a complex spike or a burst of simple spike discharge in response to the second stimulus in the paired stimulus paradigm must have resulted from the inhibition of trigeminal afferents that occurs with prior trigeminal stimulation and blinking (Pellegrini and Evinger 1995; Powers et al. 1997). Thus, the activity characteristics of blinkrelated Purkinje cell activity implied that these cells "listened" to sensory information rather than regulated trigeminal reflex blink magnitude.

If blink-related Purkinje cells have a minimal role in the modulation of normal trigeminal reflex blinking, then eliminating the modulation of blinkrelated Purkinje cell activity with blinking should not significantly alter trigeminal reflex blinks. To test this hypothesis, we inactivated the inferior olive to eliminate the complex spike and the burst of simple spike discharge associated with OOemg activity and checked for changes in OOemg evoked by a corneal stimulus (Fig. 5). A lidocaine injection into the inferior olive blocked the complex spike evoked by corneal stimulation and reduced significantly the burst of simple spike discharge correlated with the termination of OOemg activity (Fig. 5A). Following inferior olive inactivation, mean spontaneous firing rate of simple spikes increased by $13 \%$, whereas mean burst frequency fell by $11 \%$. These changes reduced significantly the burst/ spontaneous ratio from 1.467 to 1.163 ( $t$-test, $P<0.001 ; n=23$ cells). The disruption of blinkrelated Purkinje cell modulation, however, did not consistently alter OOemg activity (Fig. 5B,C). The mean change in corneally evoked OOemg magni-
Figure 5: Effect of lidocaine inactivation of the inferior olive on blink-related Purkinje cell discharge and OOemg activity evoked by corneal stimulation. (A) Histogram of simple spike responses (left) and cell activity (right) evoked by corneal stimulation for a blink-related Purkinje cell before (pre-Lidocaine) and after (Lidocaine IO) inactivating the inferior olive with a microinjection of lidocaine. The histogram is the sum of 20 trials using 10-msec bins. (B) Rectified and averaged OOemg responses evoked by 20 consecutive corneal stimuli before (solid line, Pre Lidocaine) and after (dashed line, Lidocaine $\mathrm{IO}$ ) inactivation of the inferior olive. $(C)$ Histogram of the change in corneally-evoked OOemg magnitude following inferior olive inactivation relative to OOemg magnitude before the inferior olive inactivation.

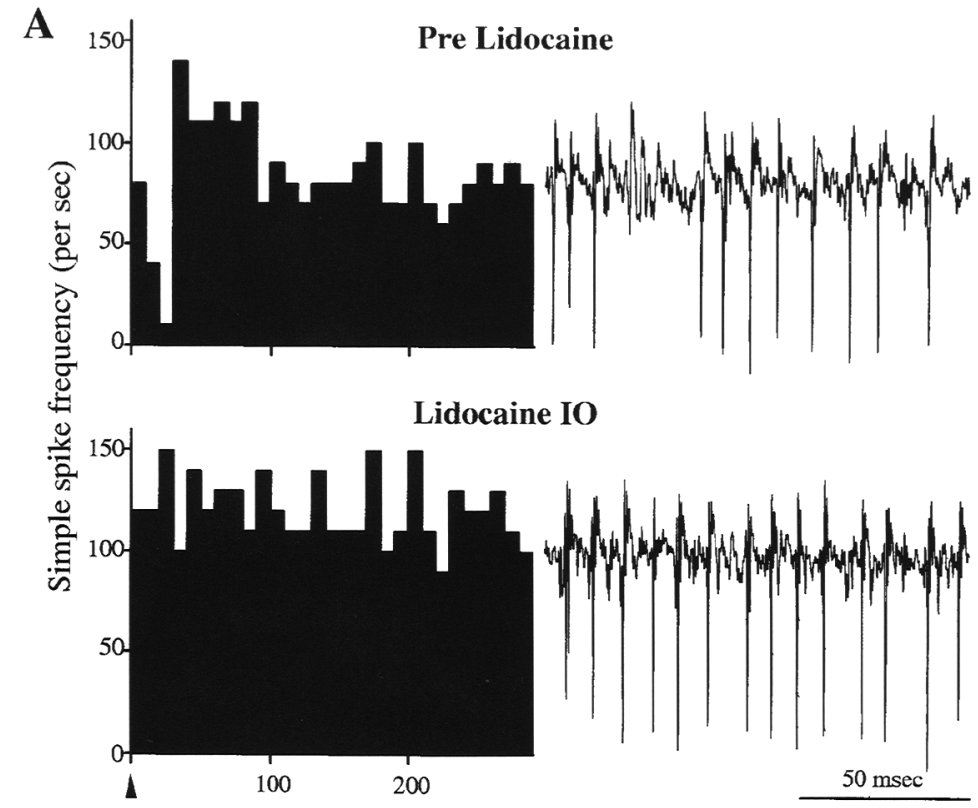

B

Stim. Time (msec)
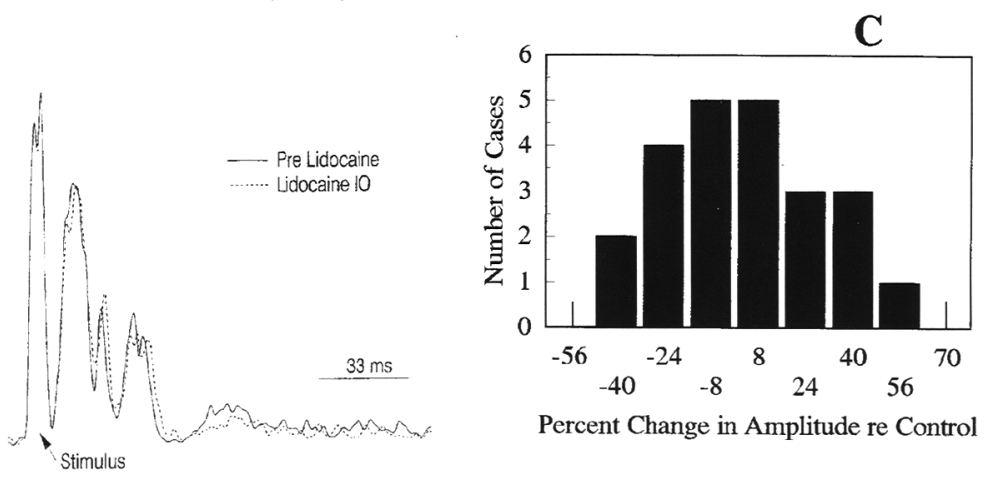

Percent Change in Amplitude re Control

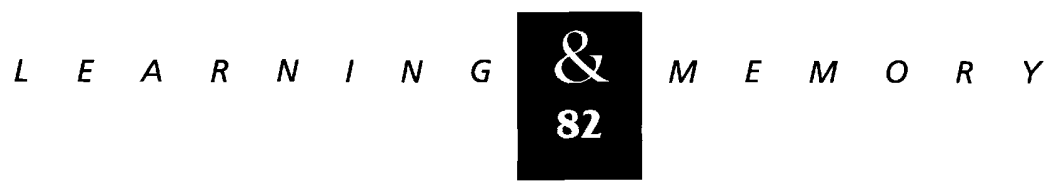


tude was an insignificant $2.9 \%$ decrease following inferior olive inactivation ( $t$-test, $P>0.25 ; n=23$ experiments). Therefore, reducing or eliminating the modulation of blink-related Purkinje cell activity evoked by corneal stimulation did not significantly alter OOemg magnitude or duration.

\section{ADAPTIVE MODIFICATION OF THE BLINK REFLEX}

A reduction in eyelid motility induces an increase in the magnitude and excitability of trigeminal reflex blinks (Evinger and Manning 1988; Evinger et al. 1989; Schicatano et al. 1996). These adaptive increases occur almost exclusively in the long latency components of OOemg activity (Evinger and Manning 1988; Evinger et al. 1989). Because this is the only segment of reflex blink activity susceptible to cerebellar influence, the cerebellum could participate in the adaptive increase in the magnitude and duration of reflex blinking. We investigated this possibility by recording the activity of seven blink-related Purkinje cells in six rats before and during lid restraint. In addition, we tested the ability of rats to produce adaptive increases of the blink reflex following cerebellar cortex lesions.

The activity of blink-related Purkinje cells correlated with OOemg activity during lid restraint (Fig. 6). In three of the seven experiments, lid restraint failed to initiate significant increases $(0 \%-$
$4 \%$ ) in reflex blink magnitude. The Purkinje cells recorded in these experiments did not exhibit significant changes in simple spike activity (Fig. 6B). In the remaining four experiments, the magnitude of OOemg activity increased from $33.5 \%$ to $112 \%$ over prerestraint values. This elevation of OOemg magnitude occurred primarily in the longer latency components of reflex blink activity and from an increase in blink duration (Fig. 6A). The blink-related Purkinje cells recorded in these experiments all exhibited a backward shift in the time of the occurrence of maximum spike density (Fig. 6C). This delay in the peak simple spike burst should have lengthened the period of interpositus neuron activity associated with the reflex blink (Fig. 2). In contrast to the shift in the timing of peak burst, the tonic simple spike firing frequency in the interblink interval did not change in the brief periods ( $<40 \mathrm{~min}$ ) that we tested lid restraint.

Another test of the role of the cerebellar cortex in adaptive modification of the blink reflex was to investigate whether adaptive blink modification occurred following a unilateral lesion of the cerebellar cortex containing blink-related Purkinje cells (Fig. 7). Because the corneal reflex blink is normally unilateral in rats (Basso et al. 1996), it is possible to assess lesion affects on the two lids independently. Three decerebrate rats were challenged with lid restraint that enhanced the amplitude and duration of OOemg activity in response to a corneal stimulus (Fig. 7B, Pre). Restraint was re-

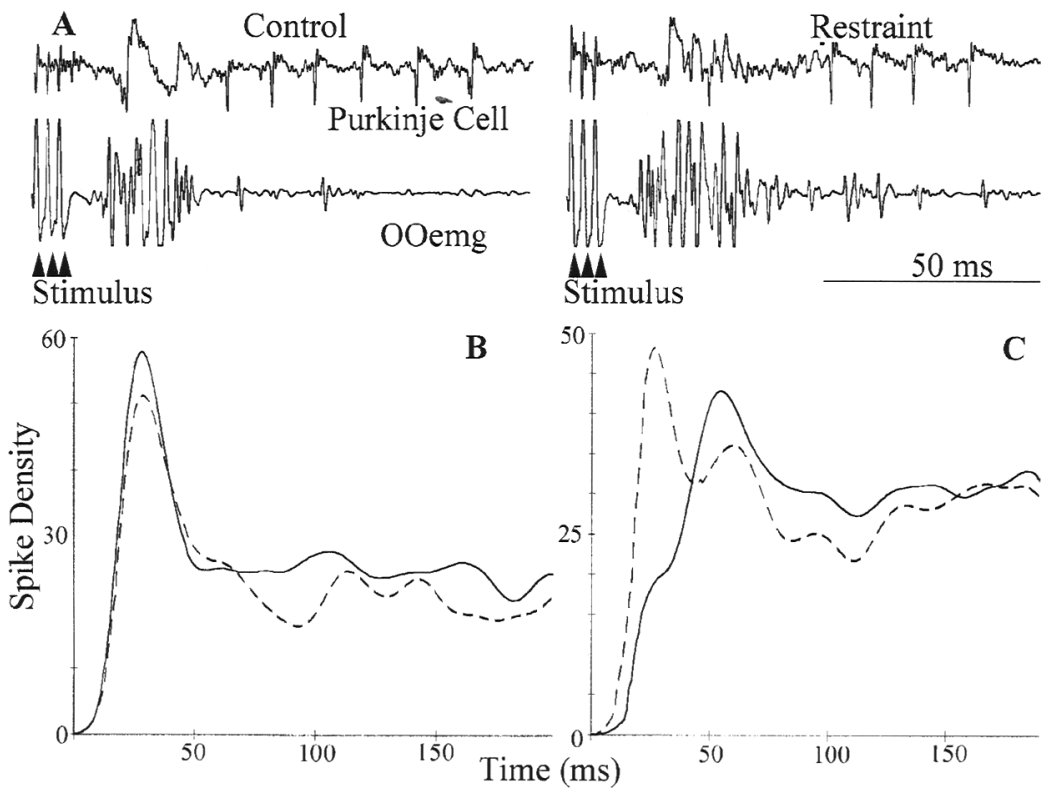

Figure 6: Effect of restraining the upper eyelid on Purkinje cell activity and OOemg responses. $(A)$ Individual records of blink-related Purkinje cell and OOemg activity in response to a corneal stimulus (arrowheads, Stimulus) before (Control) and after restraint of the upper eyelid (Restraint). (B) Spike density of simple spike activity of a single Purkinje cell before (dashed line) and during (solid line) restraint of the eye lid that did not produce an adaptive increase in OOemg activity. Each spike density record is the average of 20 trials in which $\sigma=2 \mathrm{msec}$. (C) Spike density of simple spike activity of a single Purkinje cell before (dashed line) and during (solid line) restraint of the eye lid that produced an adaptive increase in OOemg activity. Each spike density record is the average of 20 trials in which $\sigma=2 \mathrm{msec}$.

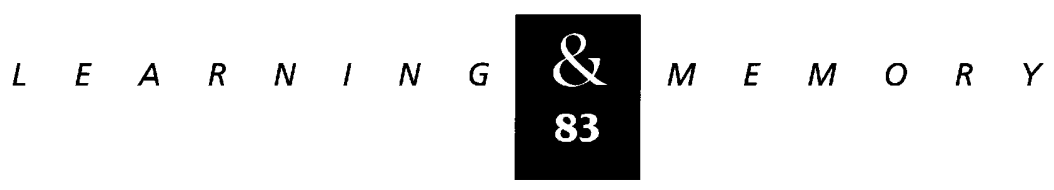


Figure 7: Effect of lesions of the left cerebellar cortex on adaptation of the corneal blink reflex. $(A)$ Reconstruction of a lesion that abolished adaptive gain increases for the left eyelid. Three schematic sections of rat cerebellum adapted from Swanson (1992) 11.4, 11.75, and $12.2 \mathrm{~mm}$ posterior to bregma. Solid areas show site of lesion. (B) OOemg amplitude relative to prerestraint OOemg values for the left $(\square, \boldsymbol{\square})$ and right $(\diamond$, -) OOemg during restraint of the eyelids before $(\square, \diamond)$ and after $(\square, \bullet)$ a lesion of the cerebellar cortex depicted in $A$. Each point is the average of five trials. (C) OOemg amplitude relative to prerestraint OOemg values for the left $(\boldsymbol{\square})$ and right $(\bullet)$ OOemg during restraint of the upper eyelids three days after a lesion of the left cerebellar cortex. Each point is the average of five trials.
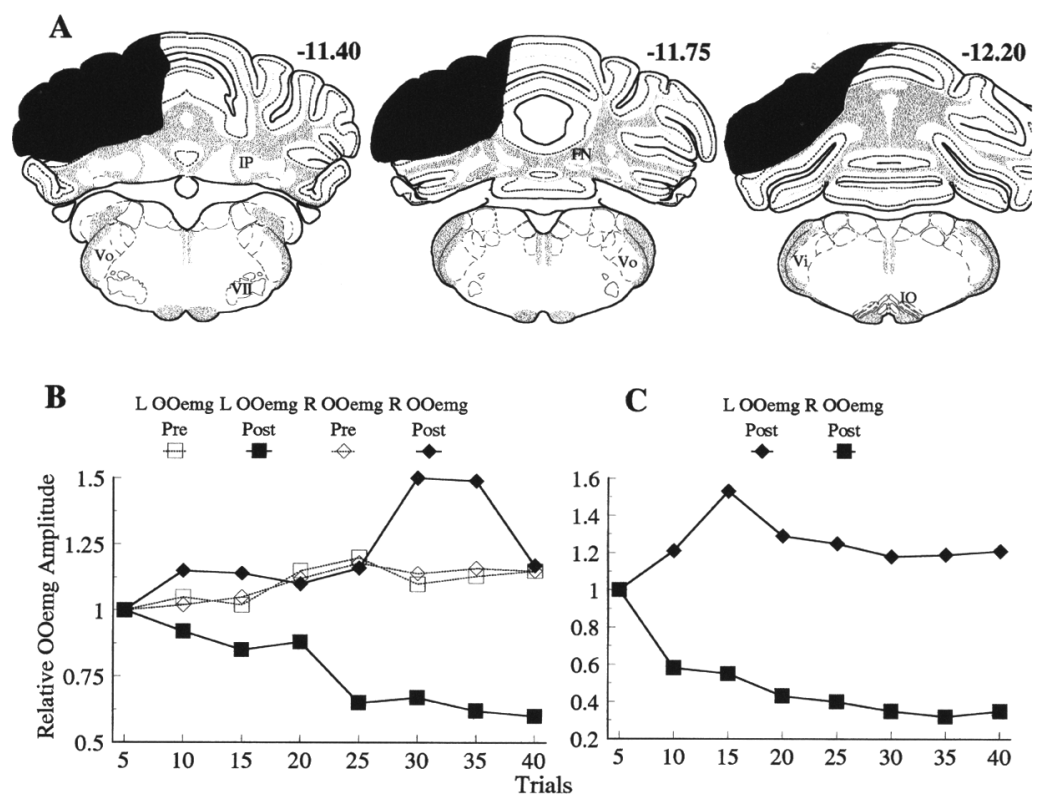

moved and OOemg activity returned to prerestraint levels (not illustrated). Aspiration in the left cerebellar cortex region containing blink-related Purkinje cells, altered the response to lid restraint in two of the rats. The right OOemg exhibited normal increases in OOemg activity, whereas the left OOemg exhibited a decrease in activity (Fig. 7B, Post). Histology revealed that the lesions in these two animals completely ablated cortical regions containing blink-related Purkinje cells (Fig. 7A). The third rat, with an incomplete lesion of the cerebellar cortex containing blink-related Purkinje cells, exhibited increases in OOemg activity nearly identical to that seen before the lesions. In another group of three rats, we performed lesions in the left cerebellar cortex three or four days before decerebration and testing of the adaptive modification of the corneal reflex blink. In the two animals where the earlier lesion completely abolished the cortical areas containing blink-related Purkinje cells, the left OOemg failed to adapt, whereas the right OOemg exhibited normal adaptation (Fig. 7C).

\section{Discussion}

\section{ROLE OF THE CEREBELLUM IN TRIGEMINAL REFLEX BLINKS}

Blink-related Purkinje cell activity can only act on the longer latency components of OOemg ac- tivity evoked by a corneal stimulus. The first Purkinje cell response to corneal stimulation, a complex spike, occurs after the initiation of OOemg activity. Likewise, the burst of spikes in rat deep cerebellar neurons is also too late to initiate OOemg activity because the burst appears to take place at the same time as the complex spike in Purkinje cells. A study of deep cerebellar nucleus neurons in cats reached an identical conclusion (Gruart and Delgado-García 1994). The burst of simple spike activity of blink-related Purkinje cells and the pause in interpositus neuron activity correlates with the termination of OOemg activity. Therefore, the cerebellum could modulate the long latency components and the duration of trigeminal reflex blinks, but the cerebellum could not influence the initiation of corneal reflex blinks.

A possible flaw in the previous arguments about the ability of the cerebellum to modulate the initiation of trigeminal reflex blinks is that changes in the tonic level of interpositus activity could provide moment-to-moment regulation of all reflex blink components. The results of inferior olive inactivation, however, argue against this possibility. As reported previously (Benedetti et al. 1983; Batini et al. 1985), inactivation of the inferior olive increases the level of tonic Purkinje cell simple spike activity (Fig. 5). This increased simple spike activity causes a decrease in deep cerebellar neuron activity and excitability. These changes in tonic activity, however, fail to modify reflex blinks. Therefore, altering cerebellar activity fails to

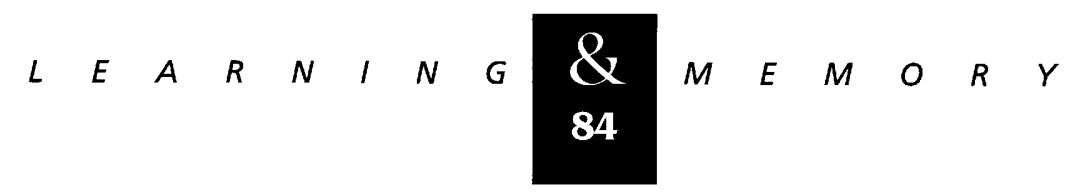


modify trigeminal reflex blinks in the short term. Previous studies of the effect of cerebellar damage on reflex blinking are conflicting. Some investigators report that cerebellar damage does not affect unconditioned blink responses (for review, see Steinmetz et al. 1992), although others find a decrement in unconditioned response magnitude (for review, see Bloedel and Bracha 1995). Yeo and Hardiman (1992) report an increase in the magnitude of unconditioned responses following cerebellar cortical lesions.

The activity of blink-related Purkinje cells appears to reflect sensory- rather than motor-related signals. The complex spike evoked in blink-related Purkinje cells is a trigeminal sensory signal, as the complex spike can be elicited in the absence of OOemg activity (Fig. 1). Likewise, the burst of simple spike activity correlated with the end of OOemg activity is absent when blinks are reduced in amplitude as occurs in the paired stimulus paradigm (Fig. 4). The reduction in the burst of simple spike activity following inactivation of the inferior olive (Fig. 5) suggests that the occurrence of a simple spike acts to gate mossy fiber trigeminal sensory signals. The paired stimulus paradigm also demonstrates that suppression occurring within the spinal trigeminal complex (Pellegrini and Evinger 1995) can eliminate evoked complex spikes in blink-related Purkinje cells as well as the burst of simple spike activity. ${ }^{1}$ If, as the current data indicate, the blink-related Purkinje cells exhibit a sensory rather than a motor signal, it is not surprising that eliminating Purkinje cell modulation fails to modify trigeminal reflex blinks in the short term (Fig. 5).

\section{ROLE OF THE CEREBELLUM IN ADAPTIVE MODIFICATION OF TRIGEMENAL REFLEX BLINKS}

Despite the evidence against a role for the cerebellum in regulating reflex blinks, the current lesion and recording data suggest a powerful role for the cerebellum in adaptive modification of trigeminal reflex blinks. This incongruity can be reconciled by assuming that trigeminal sensory signals

\footnotetext{
${ }^{1}$ Because all blinks induce a transient suppression of trigeminal inputs (Powers et al. 1997), the absence of a complex spike evoked by an unconditioned stimulus following a conditioned response may be attributable to trigeminal inhibition rather than interpositus inhibition of the inferior olive.
}

modify cerebellar circuits which, in turn, reinforce or stabilize desired synaptic changes within trigeminal reflex blink pathways. In this scheme, reorganization of trigeminal signals alter cerebellar activity. Consistent changes in trigeminal signals initiated by adaptive modification may "teach" the cerebellum a new firing pattern. This new firing pattern reinforces or stabilizes the adaptive modifications in trigeminal reflex blink circuits. Without the cerebellum, attempts to alter the gain-of-trigeminal blink reflexes fail to develop appropriately. Nevertheless, although there is no drive to alter trigeminal reflex blinks, removal of the cerebellum will not effect trigeminal reflex blinks in the short term. After prolonged period of cerebellar damage, however, we would expect the gainof-trigeminal reflex blinks to drift. This suggestion is consistent with the conflicting reports about the effect of cerebellar damage on reflex blinks in conditioned eyelid studies.

Aspiration of the cerebellar cortex containing blink-related Purkinje cells not only prevents adaptive increases in OOemg magnitude, cerebellar cortex damage leads to a decrease in OOemg magnitude with lid restraint (Fig. 7). A similar decrease in blink amplitude following lid restraint occurs after a lesion of the supraorbital branch of the trigeminal nerve which provides sensory feedback from the blink (Evinger et al. 1989). If trigeminal sensory inputs modulate the activity of blink-related Purkinje cells, it makes sense that a loss of trigeminal sensory input and elimination of blink-related Purkinje cells produce the same disruption of adaptive gain modification. The reason that blink magnitude decreases in these two conditions may be the relative weightings of inhibitory and facilitatory forces that sum to produce a reflex blink. A trigeminal blink-evoking stimulus probably initiates both a positive feedback circuit to produce the blink and a slower acting inhibitory process to terminate the blink (Evinger 1995). An adaptive increase in blink magnitude can occur by delaying or reducing this inhibitory process. If so, attempts to modify blink magnitude destabilize the inhibitory processes. When changes in the cerebellar output do not reinforce rearrangements of the inhibitory processes, they tend to drift toward higher levels, which would reduce blink magnitude. Because uncontrollable spasms of lid closure result if the inhibitory processes drift toward lower levels (Schicatano et al. 1997), the system probably has a built in tendancy to move toward more rather than less inhibition when adaptation occurs.

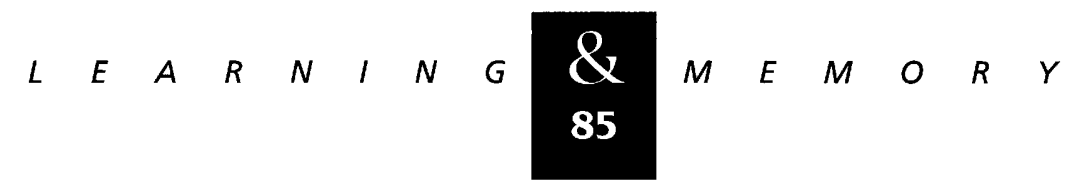


Although the current study primarily examined the cerebellar cortex, the results do not imply that the cerebellar cortex is the sole site of plasticity associated with adaptive gain changes. A recent model of the cerebellum (Mauk and Donegan, this issue) illustrates how cerebellar plasticity can be distributed between the cerebellar cortex and the cerebellar nuclei. In the initial stages of plasticity, the cerebellar cortex is crucial in teaching the deep cerebellar nuclei. Therefore, lesions of the cerebellar cortex in the initial stages of gain changes would disrupt adaptive plasticity. After adaptation had occurred, cortical lesions would exert little effect on reflex blinks, because the deep cerebellar nuclei had already undergone gain modification. The current data are completely consistent this model.

The data from the current and a previous (E.J. Schicatano, K.R. Peshori, V.M. Henriquez, and C. Evinger, unpubl.) study suggest that the trigeminal complex, rather than the cerebellum, is the pivotal site for adaptive modification of the trigeminal reflex blinks. Many studies of Pavlovian conditioning of the eye blink argue that the cerebellum is the pivotal site for acquisition of conditioned responses (e.g., Thompson and Krupa 1994). Both of these views are consistent with the current scheme of cerebellar regulation of blinking. The sensory cues necessary for adaptive modification of reflex blink magnitude, motor efference copy, and sensory feedback from the movement are both immediately available in the brainstem trigeminal complex. In contrast, the association of an auditory signal and a reflex blink necessary for the creation of a conditioned response are more readily available in the cerebellum than in the trigeminal complex. Therefore, the two paradigms rely more heavily on one portion of the circuit rather than the other. If the cerebellum is an integral component in a trigeminal reflex blink circuit, then changes in either the trigeminal system or in the cerebellum will modify other components of the circuit. The current data show how modifications occurring within the trigeminal system alter the response of blink-related Purkinje cells, as in the paired stimulus paradigm (Fig. 4). Likewise, the acquisition of conditioned responses alters the threshold and excitability of the trigeminal complex (E. Schicatano and C. Evinger, unpubl.). Therefore, the simplest interpretation of these data is that the interactions between the cerebellum and the trigeminal system are reciprocal and that both undergo plastic changes that reinforce each other. In adaptive changes, the trigeminal complex is the driving force, whereas in eye-blink conditioning, the cerebellum is the driving force.

\section{Acknowledgments}

We thank Donna Schmidt for her excellent technical assistance. This work was supported by grant EY07391 from the National Eye Institute (C.E.).

The publication costs of this article were defrayed in part by payment of page charges. This article must therefore be hereby marked "advertisement" in accordance with 18 USC section 1734 solely to indicate this fact.

\section{References}

Basso, M.A., A.S. Powers, and C. Evinger. 1996. An explanation for blink reflex hyperexcitability in Parkinson's disease. I. Superior colliculus. J. Neurophysiol. 16: 7308-7317.

Batini, C., J.M. Billard, and H. Daniel. 1985. Long-term modification of cerebellar inhibition after inferior olive degeneration. Exp. Brain Res. 59: 404-409.

Benedetti, F., P.G. Montarolo, P. Strata, and F. Tempia. 1983. Inferior olive inactivation decreases the excitability of the intracerebellar and lateral nuclei in the rat. J. Physiol. (Lond.) 340: 195-208.

Bloedel, J.R. and V. Bracha. 1995. On the cerebellum, cutaneomuscular reflexes, movement control and the elusive engrams of memory. Behav. Brain Res. 68: 1-44.

Chuke, J.C., R.S. Baker, and J.D. Porter. 1996. Bell's palsy-associated blepharospasm relieved by aiding eye closure. Ann. Neurol. 39: 263-268.

duLac, S., J.L. Raymond, T.J. Sejenowski, and S.G. Lisberger. 1995. Learning and memory in the vestibulo-ocular reflex. Annu. Rev. Neurosci. 18: 409-442.

Evinger, C. 1995. A brainstem reflex in the blink of an eye. News Physiol. Sci. 10: 147-153.

Evinger, C. and K.A. Manning. 1988. A model system for motor learning: Adaptive gain control of the blink reflex. Exp. Brain Res. 70: 527-538.

Evinger, C., J.J. Pellegrini, and K.A. Manning. 1989. Adaptive gain modification of the blink reflex. A model system for investigating the physiologic bases of motor learning. Annu. N.Y. Acad. Sci. 253: 87-100.

Evinger, C., M.A. Basso, K.A. Manning, P.A. Sibony, J.J. Pellegrini, and A.K.E. Horn. 1993. A role for the basal ganglia in nicotinic modulation of the blink reflex. Exp. Brain Res. 92: 507-515.

Gruart, A. and J.M. Delgado-García. 1994. Discharge of identified deep cerebellar nuclei neurons related to eye blinks in the alert cat. Neuroscience 61: 665-681.

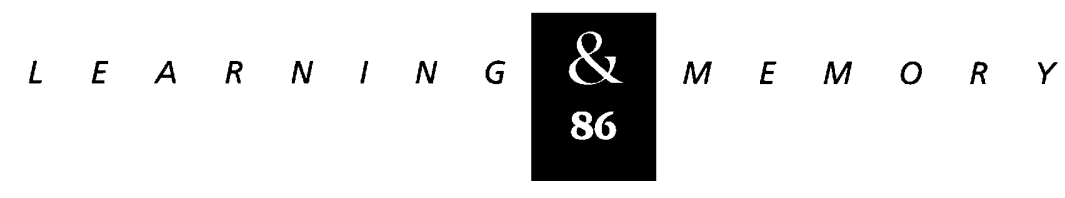


Huffman, M.D., R.S. Baker, M.W. Stava, J.C. Chuke, B.R. Rouholiman, and J.D. Porter. 1996. Kinematic analysis of eyelid movements in patients recovering from unilateral facial nerve palsy. Neurology 39: 263-268.

Mauk, M.D. and N.H. Donegan. 1997. A model of Pavlovian eyelid conditioning based on the synaptic organization of the cerebellum. Learn. \& Mem. (this issue).

Pellegrini, J.J. and C. Evinger. 1995. The trigeminally evoked blink reflex: II. Mechanisms of paired stimulus suppression. Exp. Brain Res. 107: 181-196.

Pellegrini, J.J., A.K.E. Horn, and C. Evinger. 1995. The trigeminally evoked blink reflex: I. Neuronal circuits. Exp. Brain Res. 107: 166-180.

Powers, A.S., E.S. Schicatano, M.A. Basso, and C. Evinger. 1997. To blink or not to blink: Inhibition and facilitation of reflex blinks. Exp. Brain Res. 113: 283-290.

Richmond, B.J., L.M. Optican, M. Podell, and H. Spitzer. 1987. Temporal encoding of two-dimensional patterns by single units in primate primary visual cortex. I.

Stimulus-response relationships. J. Neurophysiol.

57: 132-146.

Schicatano, E.J., M.A. Basso, and C. Evinger. 1997. An animal model explains the origins of the cranial dystonia benign essential blepharospasm. J. Neurophysiol. (in press).

Steinmetz, J.E., D.G. Lavond, D. Ivkovich, C.G. Logan, and R.F. Thompson. 1992. Disruption of classical eyelid conditioning after cerebellar lesions: Damage to a memory trace system or a simple performance deficit? J. Neurosci. 12: $4403-4426$.

Swanson, L.W. 1992. Structure of the rat brain. Elsevier, Amsterdam, The Netherlands.

Thomas, R.C. and V.J. Wilson. 1965. Precise localization of Renshaw cells with a new marking technique. Nature 206: $211-213$.

Thompson, R.F. and D.J. Krupa. 1994. Organization of memory traces in the mammalian brain. Annu. Rev. Neurosci. 17: 519-549.

van Ham, J.J. and C.H. Yeo. 1992. Somatosensory trigeminal projections to the inferior olive, cerebellum and other precerebellar nuclei in rabbits. Eur. J. Neurosci. 4: 302-317.

Yeo, C.H. and M.J. Hardiman. 1992. Cerebellar cortex and eye blink conditioning: A reexamination. Exp. Brain Res. 88: 623-638.

Received February 18, 1997; revised version accepted April 30, 1997. 


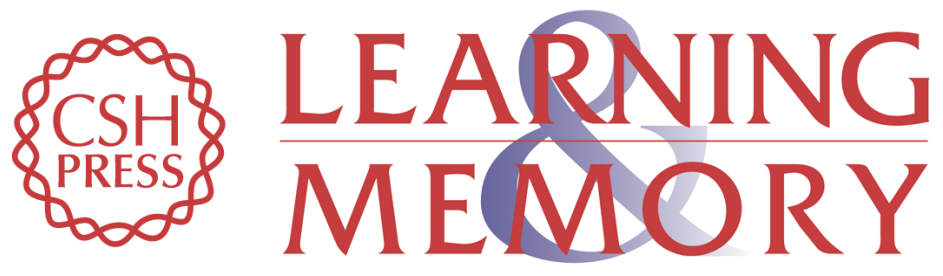

\section{Role of cerebellum in adaptive modification of reflex blinks.}

J J Pellegrini and C Evinger

Learn. Mem. 1997, 4:

Access the most recent version at doi:10.1101/lm.4.1.77

References This article cites 21 articles, 1 of which can be accessed free at: http://learnmem.cshlp.org/content/4/1/77.full.html\#ref-list-1

License

Email Alerting Receive free email alerts when new articles cite this article - sign up in the box at the Service top right corner of the article or click here. 